ARTICLE

\title{
Nanotwin-governed toughening mechanism in hierarchically structured biological materials
}

Yoon Ah Shin ${ }^{1, \star}$, Sheng Yin ${ }^{2, \star}$, Xiaoyan Li ${ }^{2,3}$, Subin Lee ${ }^{1}$, Sungmin Moon ${ }^{1}$, Jiwon Jeong ${ }^{1,4}$, Minhyug Kwon?, Seung Jo Yoo ${ }^{5}$, Young-Min Kim ${ }^{4,6}$, Teng Zhang ${ }^{2}$, Huajian $\mathrm{Gao}^{2}$ \& Sang Ho Oh¹,6

As a natural biocomposite, Strombus gigas, commonly known as the giant pink queen conch shell, exhibits outstanding mechanical properties, especially a high fracture toughness. It is known that the basic building block of conch shell contains a high density of growth twins with average thickness of several nanometres, but their effects on the mechanical properties of the shell remain mysterious. Here we reveal a toughening mechanism governed by nanoscale twins in the conch shell. A combination of in situ fracture experiments inside a transmission electron microscope, large-scale atomistic simulations and finite element modelling show that the twin boundaries can effectively block crack propagation by inducing phase transformation and delocalization of deformation around the crack tip. This mechanism leads to an increase in fracture energy of the basic building block by one order of magnitude, and contributes significantly to that of the overall structure via structural hierarchy.

\footnotetext{
${ }^{1}$ Department of Materials Science and Engineering, Pohang University of Science and Technology (POSTECH), Pohang 37673, Republic of Korea. ${ }^{2}$ School of Engineering, Brown University, Providence, Rhode Island 02912, USA. ${ }^{3}$ Center for Advanced Mechanics and Materials, Applied Mechanics Laboratory, Department of Engineering Mechanics, Tsinghua University, Beijing 100084, China. ${ }^{4}$ IBS Center for Integrated Nanostructure Physics (CINAP), Institute for Basic Science, Sungkyunkwan University, Suwon 16419, Republic of Korea. ${ }^{5}$ Nano-Bio Electron Microscopy Research Group, Korea Basic Science Institute (KBSI), Daejeon 34133, Republic of Korea. ${ }^{6}$ Department of Energy Science, Sungkyunkwan University, Suwon 16419, Republic of Korea. ${ }^{*}$ These authors contributed equally to this work. Correspondence and requests for materials should be addressed to X.L. (email: xiaoyanlithu@tsinghua.edu.cn) or to H.G. (email: Huajian_Gao@brown.edu) or to S.H.O. (email: sanghooh@skku.edu).
} 
A $s$ an important class of natural biocomposite materials, mollusk shells possess remarkable mechanical strength and toughness as a consequence of their hierarchical structuring of soft organic and hard mineral constituents through biomineralization ${ }^{1-7}$. The Strombus gigas conch shell contains a high density of nanoscale $\{110\}$ growth twins in its third-order aragonite lamellae, the basic building block of the material. Although the existence of nanotwinned aragonite has been known for decades ${ }^{8-10}$, its roles and functions in mechanical behaviours and properties of biological materials have received little attention, in spite of worldwide interests in biomimetic materials and numerous studies in recent years aimed to investigate the relationship between mechanical properties (for example, moduli, strength and toughness) and the elegant nano- and hierarchical structures of biological materials ${ }^{1-7,11}$. Various toughening mechanisms in biological materials have been proposed, including microcracking and crack bridging ${ }^{3,12}$, flaw tolerance of nanostructure ${ }^{7}$, viscoelastic deformation of organic layers $^{2,13}$ and frictional sliding of mineral platelets ${ }^{6}$. In this paper, we investigate the contribution of the inherent nanoscale twins in the conch shell to its fracture toughness at the basic building block level.

Mollusk shells usually contain more than $95 \mathrm{wt} \%$ of hard yet brittle mineral (calcium carbonate in the form of aragonite or calcite) and only a tiny fraction of soft organics. They typically exhibit a fracture toughness several orders of magnitude higher than the corresponding single crystal of pure mineral ${ }^{2,3}$, which is widely attributed to the hierarchical structures extending from nano- to macro-scales ${ }^{3}$, and the staggered arrangement of mineral and organic constituents in the material ${ }^{1,4,7}$. The giant pink queen conch shell has a three-level hierarchical structure (Fig. 1a). The basic building block of this material is its third-order aragonite lamellae that contain a high density of nanoscale $\{110\}$-oriented growth twins aligned in parallel within a single lamella ${ }^{3,8-10}$. To reveal the roles of the nanoscale growth twins, in situ fracture experiments are conducted inside a transmission electron microscope (TEM) (Supplementary Figs 1-4) to investigate the interaction between the nanotwinned microstructure and a propagating crack in real-time, and to quantitatively measure the fracture toughness. In addition, large-scale atomistic simulations and finite element modelling (FEM) are performed to explore the underlying deformation mechanisms. Combining the results from both experiments and simulations indicate that the nanotwinned microstructure plays a key role in toughening the conch shell against crack propagation.

\section{Results}

Microstructural characterization. As illustrated in Fig. 1a, the basic building block of Strombus gigas is its third-order lamellae made of nanotwinned aragonite platelets $100 \sim 400 \mathrm{~nm}$ long, $\sim 75 \mathrm{~nm}$ thick and $\sim 200 \mathrm{~nm}$ wide. Parallel stacking of the third-order lamellae forms a second-order lamella with width of $5-60 \mu \mathrm{m}$ and thickness of 5-30 $\mu \mathrm{m}$, and many second-order lamellae aggregate into a first-order lamella that is several a

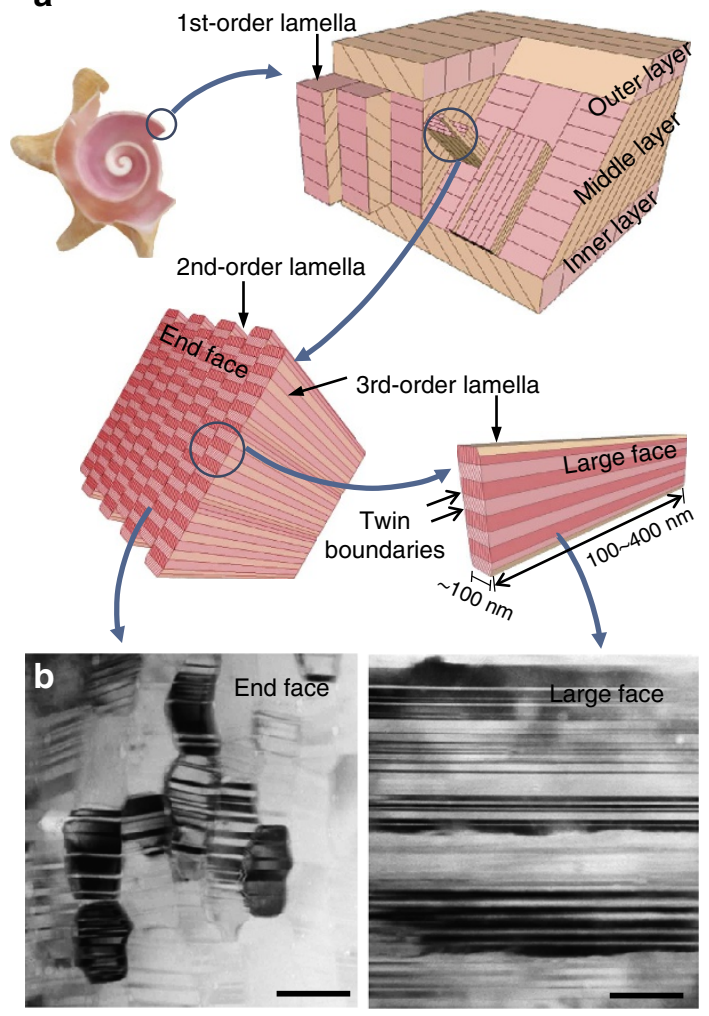

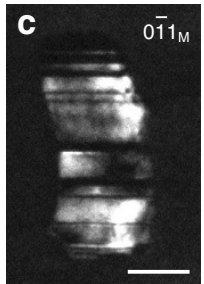
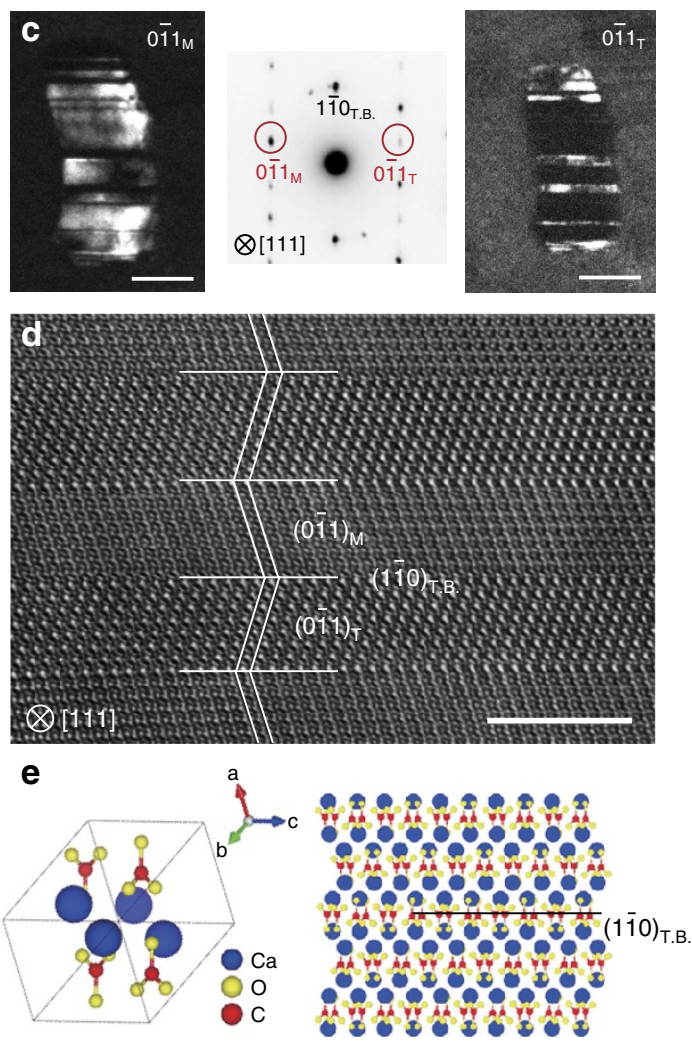

Figure 1 | Multi-level hierarchy and nanotwinned microstructure of Strombus gigas conch shell. (a) A schematic illustration of multi-level hierarchy. Refer to Supplementary Note 1 for further detailed description. (b) TEM images showing the high density of nanotwins in the third-order lamellae. The nanotwins are seen from the end-face of third-order lamellae (left of panel) and the large-face (right of panel). Scale bar, $100 \mathrm{~nm}$. (c) Dark-field TEM images of a third-order lamella taken with the $(0 \overline{1} 1)_{M}$ matrix (left of panel) and $(0 \overline{1} 1)_{T}$ twin (right of panel) reflection, and electron diffraction pattern along [111] zone axis (middle of panel). TBs are parallel to the (110) plane. Scale bar, $50 \mathrm{~nm}$. (d) HRTEM image of nanoscale twins in a third-order lamella aligned into the [111] zone axis. Scale bar, $5 \mathrm{~nm}$. (e) Orthorhombic unit cell of aragonite (left-hand side), and an atomic configuration of nanotwinned aragonite with $(1 \overline{1} 0)_{T B}$ TB (right-hand side). 
micrometres wide and 5-60 $\mu \mathrm{m}$ thick (Supplementary Fig. 5). Figure $1 \mathrm{~b}$ shows two TEM images of third-order lamellae seen from their large faces and end faces (Supplementary Fig. 6), which are surrounded by a proteinaceous matrix and tightly interlocked. Figure 1c shows two TEM dark-field images of a third-order lamella taken with the $(0 \overline{1} 1)_{\mathrm{M}}$ and $(0 \overline{1} 1)_{\mathrm{T}}$ reflections, which are related by twinning on the (110) plane, and its selected area electron diffraction pattern along the [111] zone axis. Quantitative analysis of high-resolution TEM (HRTEM) images confirms that the twin thickness varies from 2 to $20 \mathrm{~nm}$, with a mean value of $\sim 8 \mathrm{~nm}$ (Supplementary Fig. 7). A HRTEM in Fig. 1d indicates that the $(1 \overline{1} 0)_{\mathrm{TB}}$ twin boundaries (TBs) are coherent and free of defects. Figure 1e illustrates the orthorhombic unit cell of aragonite and corresponding atomic configuration of a TB. The lattice rotation between twin and matrix across the $(1 \overline{1} 0)_{\mathrm{TB}}$ measured by geometrical phase analysis (GPA) is about $36^{\circ}$ (Supplementary Fig. 8; Supplementary Note 2). The microstructural and crystallographic characterizations summarized in Fig. 1 reveal that the Strombus gigas conch shell is constructed with nanotwinned aragonite platelets as the basic building block and as such can be regarded as a nanotwinned material synthesized by nature.
In situ TEM fracture testing. In situ real-time TEM fracture experiments based on a nanoindention were used to investigate crack propagation in the nanotwinned aragonite (extracted from the conch shell) and its single-crystalline (twin-free) counterpart, and to quantitatively determine the fracture toughness by measuring crack tip opening displacement (CTOD). Specially designed nanomechanical testing specimens for CTOD measurement are shown in Fig. 2a,b, where a flat diamond nanoindenter moves downward at a constant displacement rate of $3 \mathrm{~nm} \mathrm{~s}^{-1}$ to initiate a crack from a pre-introduced notch. Details of the TEM sample design and preparation using a focused ion beam (FIB) are given in the 'Methods' section and Supplementary Methods. Figure $2 c, d$ present a sequence of TEM images of crack propagation in the nanotwinned and single-crystalline aragonites, respectively. For the single-crystalline aragonite, it is observed from Fig. $2 \mathrm{~d}$ that the main crack propagates rapidly along a cleavage plane (Supplementary Movie 2), and a few deformation twins form near the crack tip or some other stress-concentration sites (Supplementary Movie 3). However, for the nanotwinned aragonite, the main crack propagates nearly perpendicular to the TBs and is then highly deflected by the TBs, as shown in Fig. 2c (Supplementary Movie 1). More detailed TEM images
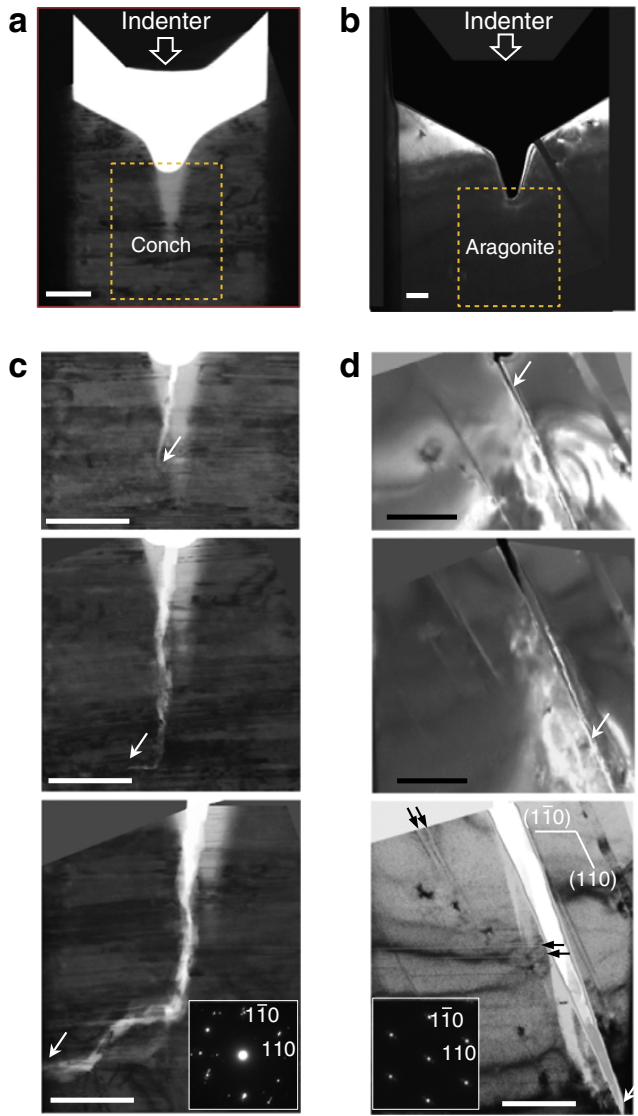
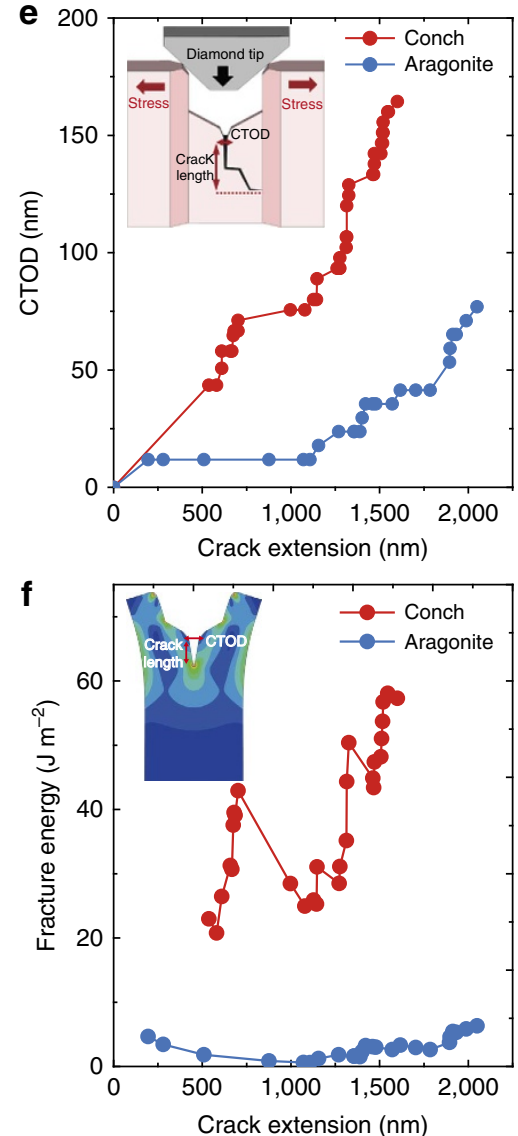

Figure 2 | Nanoscale toughness measurement by in situ TEM. (a,b) TEM samples of conch shell and aragonite single crystal prepared using FIB for nanoscale toughness measurement. A flat diamond nanoindenter moves downward at a constant displacement rate $\left(3 \mathrm{~nm} \mathrm{~s} \mathrm{~s}^{-1}\right)$ to initiate a crack at the notch. Details of TEM sample design and preparation are given in the Supplementary Methods. Note that dark-field TEM image is seen in $\mathbf{b}$ and bright-field TEM image is seen in a. Scale bar, $500 \mathrm{~nm}$. (c,d) A sequence of TEM snapshots (see Supplementary Movies 1 and 2) showing the crack propagation in the conch shell and aragonite single crystal. The crack tip is marked by white arrow in each image. The black arrows indicate the deformation twins formed along the (110) and (110) cleavage planes during the crack propagation in aragonite. The inset shows a [001] zone axis selected area electron diffraction pattern for each sample, indicating that the loading direction was parallel to the [110] direction in both the samples. Scale bar, $500 \mathrm{~nm}$. (e) CTOD as a function of the crack extension measured from in situ TEM. The inset is a conceptual representation about the measurement of CTOD and crack extension. More details are given in the Supplementary Methods. (f) Fracture energy versus crack extension from FEM. The inset shows a simulated sample used for FEM. 
(Supplementary Fig. 9) of the advancing crack tip show that the main crack can be temporarily arrested by multiple TBs, accompanied by the nucleation of a number of nanocracks ahead of its tip (Supplementary Movie 4). In contrast to the singlecrystalline aragonite, the fracture path in nanotwinned aragonite is substantially rough and curved due to the blocking and deflection by the TBs.

To quantify the fracture resistance in these two specimens, we measured the CTOD based on the $\delta_{5}$ definition $^{14}$. The details of in situ TEM measurement of CTOD are given in Supplementary Methods and Supplementary Fig. 4. In Fig. 2e, the measured CTOD is plotted against the crack extension distance. The crack in nanotwinned specimen exhibits a much larger CTOD than that in the single-crystalline specimen during extension. This suggests that the crack tip in nanotwinned aragonite becomes relatively blunt due to local plastic deformation, whereas the crack tip in singlecrystalline aragonite remains sharp, as typical for brittle fracture. To determine the fracture toughness of the material, we also performed FEM to calculate the crack-tip J-integral (representing energy released per unit fracture surface area) following the same loading and geometrical conditions as in the experiments (see 'Methods' section). The J-integral versus crack extension curve in Fig. 2f indicates that the fracture energy of the biogenic nanotwinned aragonite is about one order of magnitude larger than that of the twin-free aragonite single crystal. These results demonstrate that the pre-existing growth twins play an essential role in enhancing the fracture toughness of the conch shell.

Furthermore, we investigated the crack propagation path in twin-free biogenic aragonite specimen prepared from nacre, and found that the nanotwinned aragonite from conch shell has much higher fracture resistance (Supplementary Note 3, Supplementary Fig. 9 and Supplementary Movie 5). This is particularly interesting because nacre contains $5 \mathrm{wt} \%$ protein while the conch shell contains only $1 \mathrm{wt} \%$ protein. Previous experimental studies on the fracture of mollusk shells have shown that the soft organic phase plays a dominant role in preventing crack propagation ${ }^{2,15}$. However, the conch shell, known as the most highly mineralized mollusk shell, has less volume/weight fraction of organics but higher fracture toughness than nacre. This is consistent with our in situ TEM observations and analysis in suggesting that the nanoscale growth twins are critical in allowing the conch shell to achieve an extraordinarily high fracture toughness with minimal organic content.

Nanotwin-governed toughening mechanisms. To identify the underlying deformation mechanisms, the microstructural changes near the crack tip were investigated carefully by using HRTEM after the in situ TEM fracture experiments. In addition, dedicated in situ TEM nanoindentation experiments were carried out to observe the deformation mechanisms in real-time (Supplementary Fig. 2, and Supplementary Movies 6 and 7). Figure $3 \mathrm{~b}$ shows a representative TEM image of an advancing crack tip in the nanotwinned aragonite, which is temporarily arrested by a pre-existing TB, indicating that TBs can effectively block the crack propagation. Meanwhile, a nanocrack is nucleated and links up with the main crack, leading to crack branching, as illustrated in Fig. 3a. The influence of nanocracks on fracture toughness is discussed in detail in the context of Fig. 4. The HRTEM images in Fig. 3c,d further reveal that there exists a mixture of amorphous and nanocrystalline phases around the crack tip. Such amorphization and formation/re-orientation of nanograins are induced by the elevated stress field in the vicinity of the crack tip (Supplementary Movie 7). It indicates that when a crack is trapped by a TB, the region around the crack tip can undergo severe plastic deformation. This is further demonstrated by apparent crack blunting in Fig. 3d. For the single-crystalline aragonite, deformation twinning is found to be operative near the crack, as revealed by Figs 2d and 3e,f (Supplementary Movies 2 and 6). Some deformation twins are captured in Fig. 3f, and the corresponding twin-matrix crystallographic relation is shown in the HRTEM image (Fig. 3g). The TEM diffraction patterns show that $\{110\}$-type deformation twinning is a dominant mode in single-crystalline aragonite (Supplementary Figs 10-11). Although deformation twinning can dissipate energy to some extent, it cannot effectively hinder crack propagation, as evidenced by the sharp crack tip in Fig. $3 \mathrm{~h}$ and the catastrophic cleavage fracture shown in Fig. 2d (also see Supplementary Movies 2 and 3). A HRTEM examination shows that no phase transformation occurs near the crack tip in the single-crystalline aragonite (Fig. 3h). The differences in crack-tip deformation modes between nanotwinned and single-crystalline aragonite indicate that the pre-existing TBs of growth twins in nanotwinned biogenic aragonite are uniquely effective in blocking crack advance and enhancing energy dissipation via phase transformations in the vicinity of the crack tip, leading to higher fracture toughness shown in Fig. 2e,f.

Nanocrack toughening. More detailed TEM observations reveal that a few nanocracks are formed in the vicinity of the primary crack tip with a distinct pattern (Fig. 4a-d; Supplementary Movie 4). Here we also evaluated the contributions of multiple nanocracking to the toughness using coarse-grained simulations based on a triangular lattice model (see Supplementary Methods). In the coarse-grained simulations, the crack period $s$ varies from 300 to $1,000 \mathrm{~nm}$, whereas the crack length $2 a$ and spacing $d$ remain constant at the observed values $(2 a=200 \mathrm{~nm}$ and $d=200 \mathrm{~nm}$ ). Two simplified model patterns of nanocracks were considered, one perpendicular (referred to as $y$-nanocracks) and the other parallel ( $x$-nanocracks) to the primary crack (Fig. $4 \mathrm{e}$ ). The simulations show that the nanocracks, while reducing the elastic modulus, enhance the toughness of the material by a factor from 1.3 to 3 by shielding the main crack compared with the homogenous material (Fig. 4f), which is consistent with previous theoretical predictions ${ }^{16-19}$. More interestingly, an optimal crack pattern (indicated by an arrow in Fig. $4 \mathrm{f}$ ) yielding the maximum toughness from the current simulations for $y$-nanocracks $(s / 2 a=2.0)$ is very close to that observed in the current in situ TEM experiments (Fig. 4a-d; Supplementary Movie 4). More details of the simulations and the relevant results are given in Supplementary Methods, Supplementary Note 4 and Supplementary Figs 12-14. A combination of the above experimental measurement and the results from the coarse-grained simulations suggests that it is the crack-blocking effect of TBs, rather than multiple nanocracking, that plays the dominant role in the toughening of conch shell.

Atomistic simulations of crack propagation. To gain further insights into the atomistic mechanisms of how the nanotwinned microstructure enhances fracture resistance, we have performed large-scale molecular dynamics (MD) simulations of crack propagation in both nanotwinned and twin-free aragonites. The nanotwinned sample used in our simulations has the TB spacing of $10 \mathrm{~nm}$ as in the conch shell (Supplementary Movie 8). The sample with TB spacing of $20 \mathrm{~nm}$ was also tested to check the twin size effect on fracture toughness (see Supplementary Note 5, Supplementary Fig. 15 and Supplementary Movie 9). In these nanotwinned samples, a crack is created on the (010) plane along the [100] direction, a cleavage direction of aragonite, which is inclined with respect to the $(1 \overline{1} 0)_{\mathrm{TB}}$. For the twin-free single-crystalline aragonite, we constructed two typical samples: 


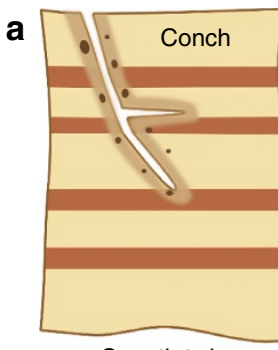

Growth twin
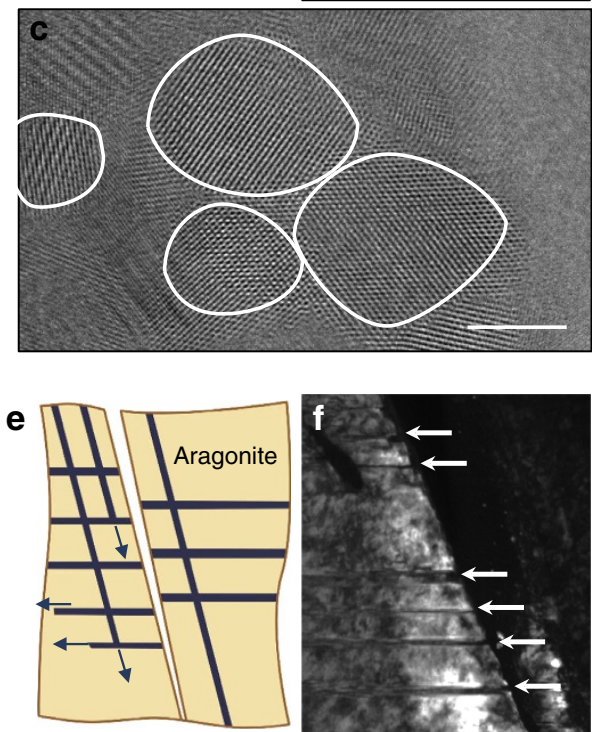

Deformation twin
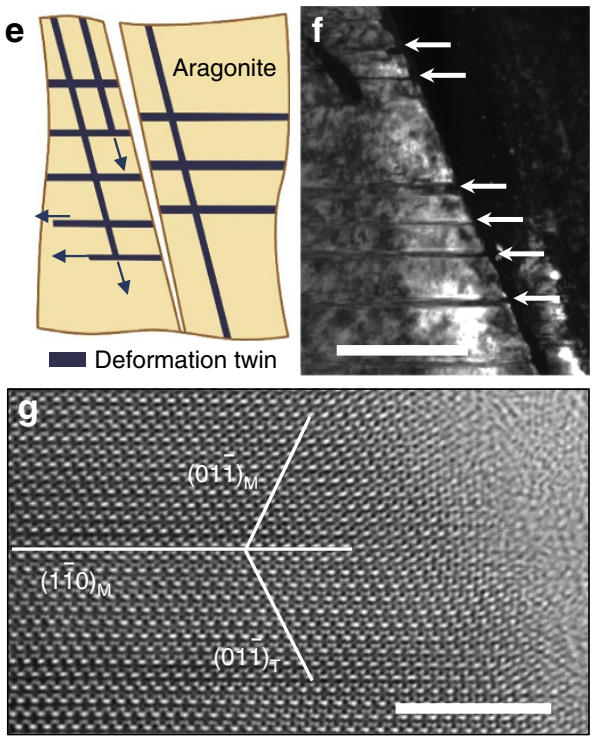
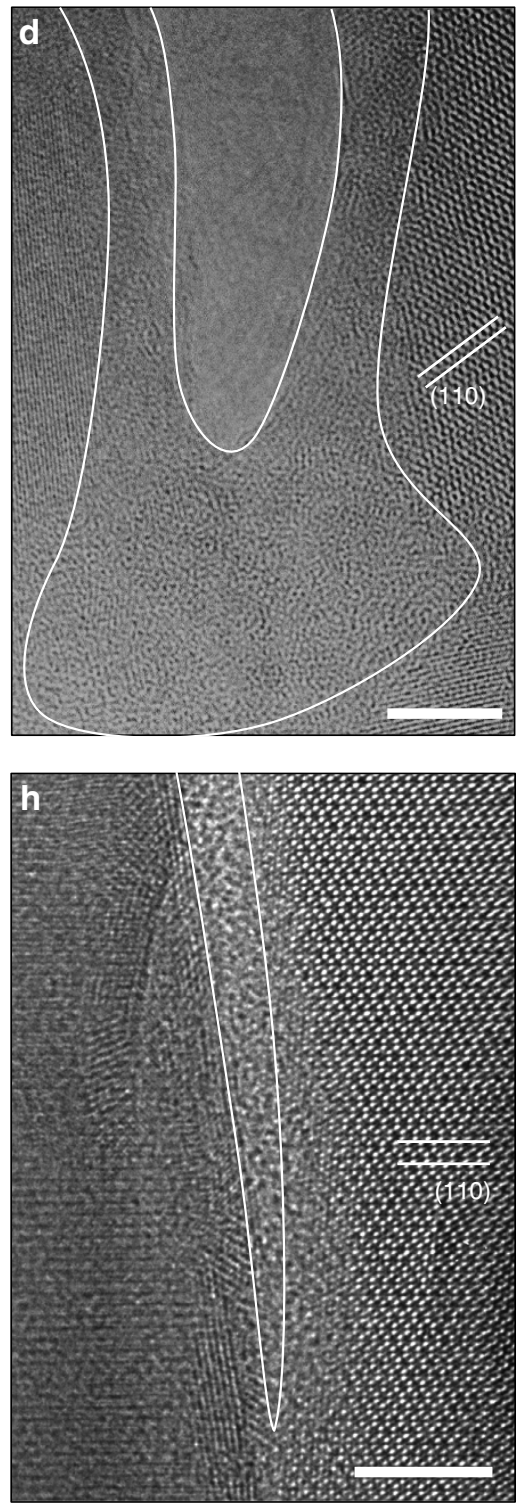

Figure 3 | Nanotwin-governed toughening mechanisms observed in experiments. (a) Schematic illustration of toughening mechanisms in the nanotwinned aragonite of conch shell, including crack-tip branching, blunting and phase transformation. The formation of amorphous and nanograins are highlighted as different colour along the periphery of the crack. (b) TEM image showing a crack tip arrested by nanoscale growth twins in the conch shell. Scale bar, $50 \mathrm{~nm}$. (c,d) HRTEM images of the edge of fractured surface of the conch shell. Some of the nanograin and amorphous phases are outlined by white lines in $\mathbf{c}$ and $\mathbf{d}$, respectively. Scale bar, $5 \mathrm{~nm}$. (e) Schematic illustration of crack tip plasticity (deformation twinning) in single-crystalline aragonite. The deformation twins are formed along the (110) and (110) planes. (f) TEM image of a typical crack in single crystal aragonite. Some deformation twins nucleated from crack tip are indicated by white arrows. Scale bar, $50 \mathrm{~nm}$. (g) HRTEM image of a deformation twin formed along the (11̄0)plane. Scale bar, $5 \mathrm{~nm}$. (h) HRTEM image of crack tip in single-crystalline aragonite. No phase transformation is observed along the atomically cleaved fracture surfaces. Scale bar, $5 \mathrm{~nm}$.

one has the same crystallographic orientation as the twin domain (Fig. 5a and Supplementary Movie 10), and the other has the orientation of the matrix (Fig. 5b and Supplementary Movie 11), which are conveniently referred to as samples with $\mathrm{T}$ - and $\mathrm{M}$-orientations, respectively. More details of the simulations are provided in the 'Methods' section.

In the twin-free aragonite with $\mathrm{T}$ - and M-orientations, deformation is localized in the vicinity of the crack tip, and the crack propagates smoothly, as evidenced by Fig. 5a,b. In contrast, the nanotwinned aragonite exhibits a very distinct fracture behaviour; the crack is always trapped at TBs for some periods of time and the crack tip becomes blunted, due to sliding along the TB (Fig. 5c,d and g), which are very similar to the experimental observations (Figs $2 \mathrm{c}$ and $3 \mathrm{~b}-\mathrm{d}$ and Supplementary Fig. 9a-f). The in-plane stress contours in Fig. $5 c$,d further reveal substantial stress/strain delocalization (alternating stresses with opposite signs) around the crack tip. These phenomena are also revealed by the FEM simulations (see Supplementary Methods and Supplementary Fig. 16). The results from both atomistic and FEM simulations demonstrate that TBs can act as effective obstacles to crack propagation, leading to crack blunting and delocalization of deformation, which facilitates energy dissipation and delays catastrophic crack propagation.

Our atomistic simulations not only showed phase transformation near the crack tip (Fig. 5f,g), but also revealed the detailed mechanism involving a coordinated rotation of some 

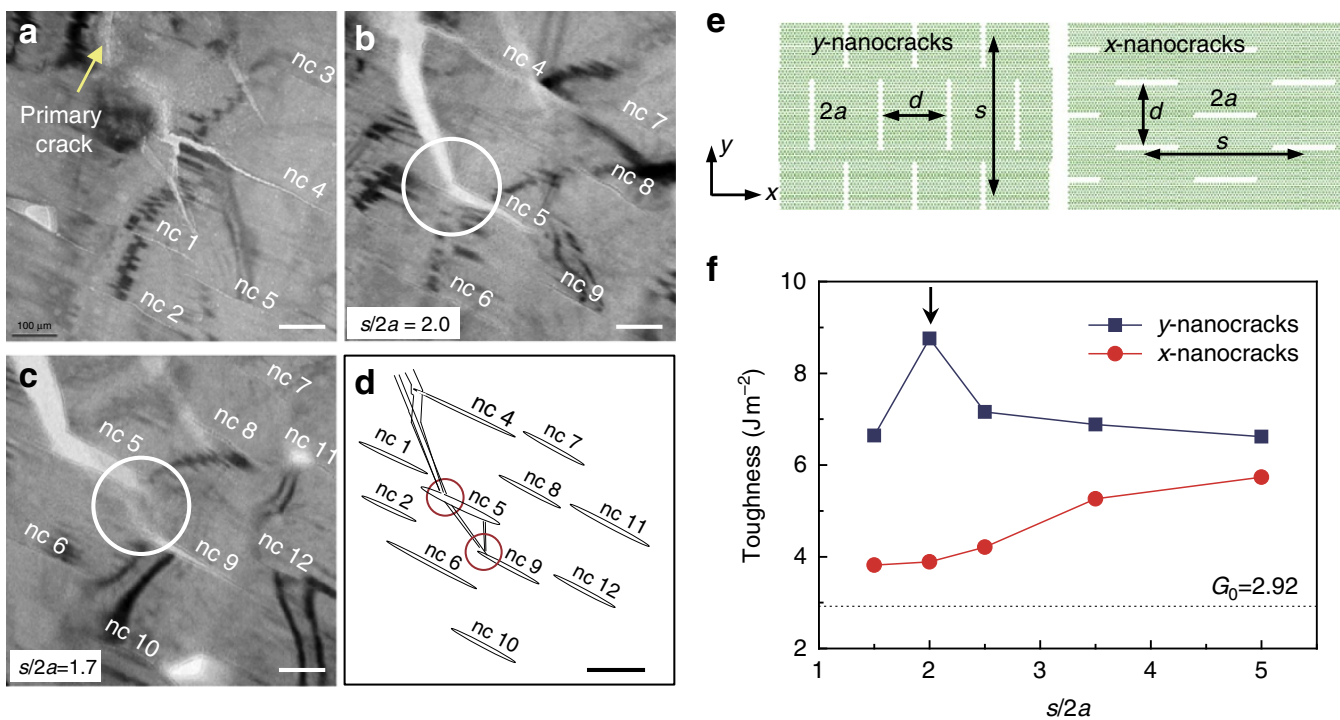

Figure 4 | Nanocrack toughening. (a) Initiation of a primary crack inclined to TBs on large faces and formation of multiple nanocracks (indicated as 'nc' followed by number) which span over hundreds of nanometres in the vicinity of the primary crack. Scale bar, $100 \mathrm{~nm}$. (b,c) TEM image showing the propagation of the primary crack through multiple nanocracking (for example, nc 5 and nc 9), resulting in an increased resistance against catastrophic propagation (Supplementary Movie 4). Scale bar, $100 \mathrm{~nm}$. (d) Schematic illustration of the nanocrack pattern in a-c. Scale bar, $100 \mathrm{~nm}$. (e) Simplified model patterns of nanocracks used in the coarse-grained simulations: one perpendicular (referred to as $y$-nanocracks) and the other parallel ( $x$-nanocracks) to the primary crack. The parameters used to describe the nanocrack pattern are: $d$ for crack spacing, $2 a$ crack length and $s$ crack period. (f) Variation of toughness with a dimensionless parameter $s / 2 a$.

a

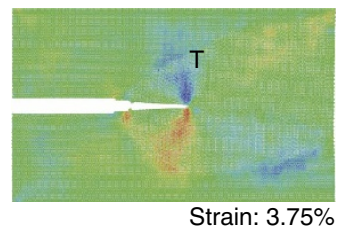

b

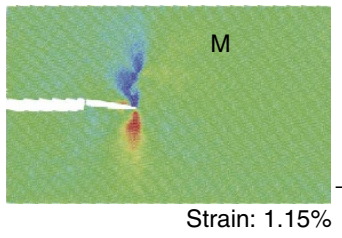

f

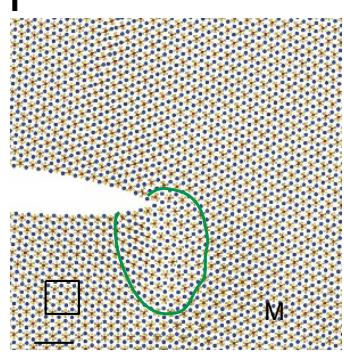

c

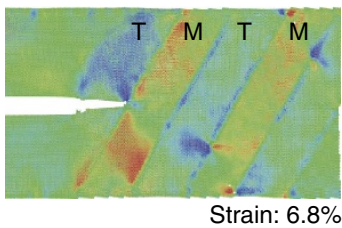

d

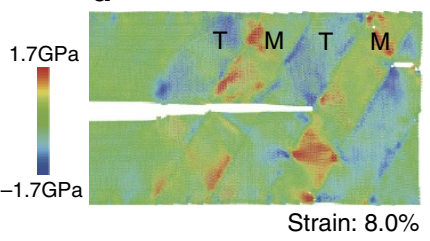

g

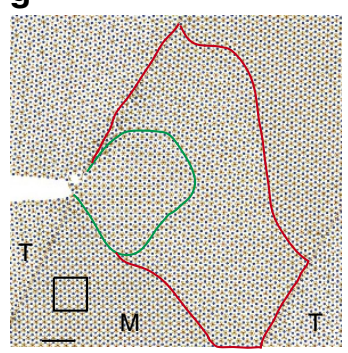

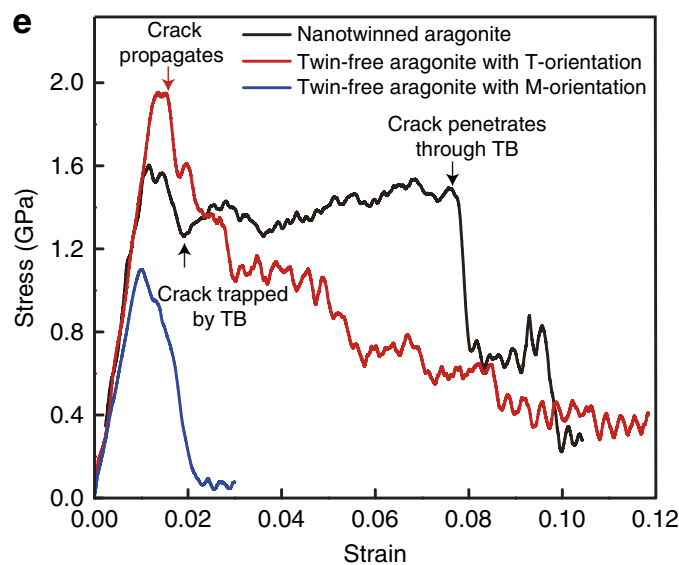

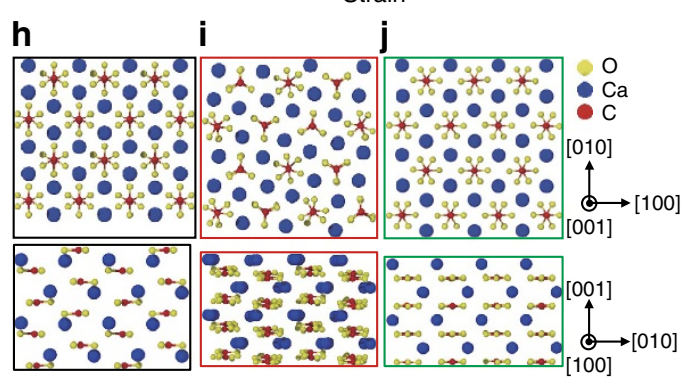

Figure 5 | Atomistic simulations of crack propagation in nanotwinned and twin-free aragonites. (a,b) Snapshots of crack propagation in twin-free aragonite single crystals with different orientations under strains of $3.75 \%$ and $1.15 \%$, respectively. The two orientations, denoted by ' $\mathrm{M}$ ' and ' $\mathrm{T}$ ', are related by (110) twin relationship. (c,d) A sequence of snapshots of crack propagation in a nanotwinned aragonite under strains of $6.8 \%$ and $8.0 \%$, respectively. (e) Stress-strain curves of nanotwinned and twin-free aragonites with the edge crack. (f,g) Structural transformation at the crack tip in single-crystalline and twinned aragonite. The perfect matrix regions are outlined by black lines, while the transformed regions are outlined by green and/or red lines. Scale bar, $2 \mathrm{~nm}$. (h-j) Typical atomic configurations of aragonite near crack tip before and after structural transformation. The pictures in $\mathbf{h}-\mathbf{j}$ illustrate the atomic structures of the regions outlined by black, red and green lines in $\mathbf{f}$ and $\mathbf{g}$, respectively. The atomic structures in upper panel are seen from the [001] direction while those in lower panel are seen from the [100] direction. 
carbonate groups $\left(\mathrm{CO}_{3}\right)$ over the transformed region driven by the crack-tip stress field, as can be seen from the typical atomic configurations of aragonite before and after the transformation (Fig. $5 \mathrm{~h}-\mathrm{j}$ ). Such phase transformation is irreversible, that is, the transformed regions cannot spontaneously recover upon unloading. Similar stress-driven phase transformation was also observed at the crack tip of nanotwinned aragonite under the nanoindenter (Fig. 3c,d) and in previous MD simulations of calcite deformation under high pressure ${ }^{20}$. It is noted that in the single-crystalline aragonite, the phase transformation occurs before crack initiation, and the transformed region is highly limited (Fig. 5f, Supplementary Note 6, Supplementary Figs 18,19 and Supplementary Table 1). In contrast, for the nanotwinned aragonite, large-scale phase transformation is activated once the crack is trapped by a TB and spreads through the nanoscale twins (Fig. $5 \mathrm{~g}$ ), resulting in delocalization of plastic deformation.

Figure $5 \mathrm{e}$ shows the stress-strain curves from our present simulations of nanotwinned and twin-free aragonites. The plateaus in the stress-strain curve of nanotwinned aragonite correspond to the crack being trapped by TBs. On the basis of the calculations in Supplementary Methods, the fracture energies of twin-free aragonites with $\mathrm{T}$ - and $\mathrm{M}$-orientations are 0.55 and $0.28 \mathrm{~J} \mathrm{~m}^{-2}$, respectively. Remarkably, the fracture energy of the nanotwinned aragonite with alternating $\mathrm{T}$ - and $\mathrm{M}$-orientations is found to be around $5.13 \mathrm{~J} \mathrm{~m}^{-2}$, which is approximately an order of magnitude higher than that of the twin-free aragonite. This dramatic enhancement in fracture toughness of nanotwinned aragonite is consistent with our experimental measurements, and the simulation results provide a strong evidence that the toughening effect originates from TBs impeding crack propagation and inducing delocalized plastic deformation. In addition, we have also conducted MD simulations of crack propagation in nanotwinned samples with TBs parallel to the initial crack (Supplementary Note 7 and Supplementary Fig. 20). The results indicate that the presence of TBs also significantly increases the fracture toughness of aragonite even if the TBs are parallel to the initial crack. This suggests that the relative orientation between the TBs and crack is a factor that affects the fracture resistance, and points to the importance of crossed lamellar stacking in upper level hierarchy (that is, first- and second-order lamellae) in hindering crack propagation from different directions.

Phase transformation in nanotwinned aragonite. For man-made nanotwinned metals, the migration of pre-existing TBs due to nucleation and slip of partial dislocations on TBs is a softening mechanism that significantly contributes the ductility of material ${ }^{21-23}$. However, for the nanotwinned aragonite from conch shells, our current in situ TEM observations (Supplementary Movie 7) showed that the preexisting (110) TBs do not migrate during nanoindentation. Figure $6 \mathrm{a}-\mathrm{c}$ showed a sequence of TEM images of the structural evolution of nanotwinned aragonite during nanoindentation, indicating that some nanoscale twins under an indenter are gradually destroyed, but TBs do not pronouncedly migrate during loading. HRTEM image and associated FFT pattern in Fig. 6d revealed that the damage of nanoscale twins is associated with the structural transformation in severe local deformation zone. This can be understood as a consequence of the change of atomic arrangement due to the rotation of carbonate groups under the local high stress level, as illustrated in Fig. $5 \mathrm{~h}-\mathrm{j}$. In the current study, inactivation of the migration of pre-existing TBs might be due to two factors. First, TBs are nearly perpendicular to the loading direction, so that there is not enough resolved shear stress to drive TB migration. Second, the strong ionic bonding structure might give rise to good stability of nanoscale twins and high critical stress for dislocation nucleation on pre-existing TBs.

\section{Discussion}

Previous theoretical models ${ }^{24-26}$ have predicted that the fracture toughness of self-similar hierarchical materials increases exponentially with the number of hierarchical levels, and this has also been demonstrated by experimental and computational studies $^{27,28}$. To further validate/complement the results from both experiments and simulations, we have extended a theoretical model of the self-similar hierarchical materials ${ }^{26}$. The relevant details of theoretical model are supplied in Supplementary Note 8. According to a bottom-up design route of the self-similar model $^{26}$, an optimal three-level hierarchical structure, referred to as a conch-like material, is built by determining the geometry and mechanical properties (including the stiffness, strength and toughness) of all the levels. We evaluated the contribution of nanoscale twins in the third-order lamellae to the overall fracture toughness of the conch-like hierarchical structure. As shown in Supplementary Fig. 17c, the overall toughness of the conch-like hierarchical material increases dramatically as the lowest-level mineral fracture energy is enhanced. Supplementary Fig. 17d provides a direct prediction that if the fracture toughness and strength of the lowest-level structure are increased only two to three times, the overall toughness of the hierarchical material will increase by one order of magnitude. These results indicate that the lowest-level structure plays a crucial role in increasing the overall toughness of the conch-like hierarchical material by driving energy dissipation in the organic matrix at all the upper levels.

Our experiments and simulations have shown that nanoscale twins enhance the fracture energy of the lowest-level structure in the aragonitic shell of Strombus gigas by about one order of magnitude. In comparison, the overall fracture energy of the conch shell is known to be about three orders of magnitude higher than that of the single-crystalline aragonite. Together with the above theoretical analysis, we conclude that the nanotwintoughened-third-order-lamellae are able to drive larger scale energy dissipation in the second- and first-order lamellae structures, leading to an exponential increase in the overall fracture energy of the material as we move up in hierarchy, in agreement with the existing theoretical models on self-similar hierarchical materials.

Previous experimental studies ${ }^{29-31}$ have shown that deformation twinning is a controlling mechanism during plastic deformation of geological aragonite and calcite crystals. In our current study, deformation twinning is observed to be a dominant energy-dissipation mechanism during crack propagation of twinfree single-crystalline aragonite, as evidenced by Figs $2 \mathrm{~d}$ and $3 \mathrm{e}-\mathrm{h}$. In contrast, in the case of biogenic nanotwinned aragonite in the conch shell, our study based on both experiments and atomistic simulations has shown that no additional twinning was activated during the fracture. Rather, it is TBs' blocking crack and phase transformation (including amorphization and formation/ reorientation of nanograins) that act as the controlling mechanism of toughening. The inactivation of deformation twinning in biogenic nanotwinned aragonite is attributed to the presence of nanoscale pre-existing twins, which apparently have suppressed the formation of additional twins near a crack tip.

Notably, there are many different species of mollusk shells in nature that exhibit different toughening mechanisms. Some of the bivalve shells, such as the brachiopod shells and Placuna placenta shells, possess more than 90 wt\% calcitic mineral which, like nacre, is free of twins. A very recent study on nanoindentation of Placuna placenta shell ${ }^{32}$ has shown that profuse deformation twinning 
a

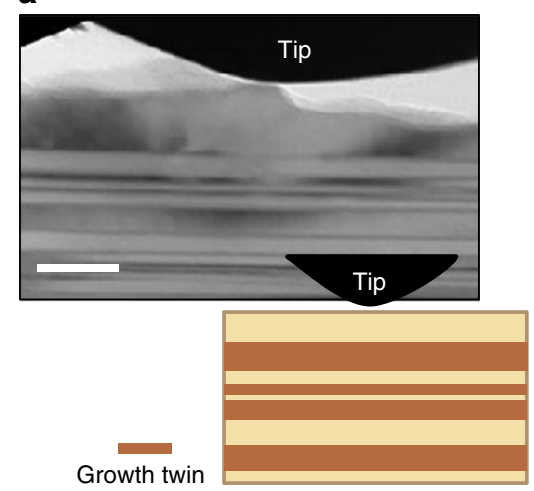

b

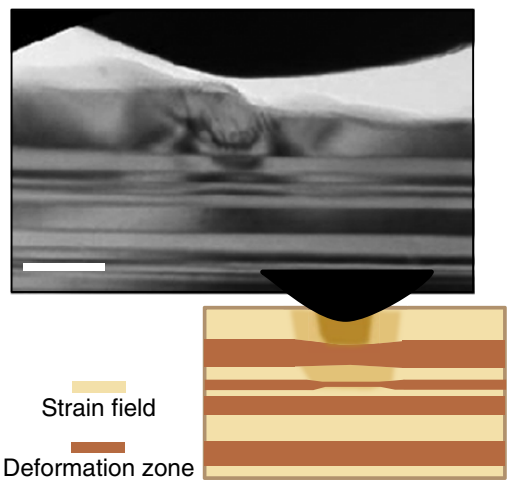

c

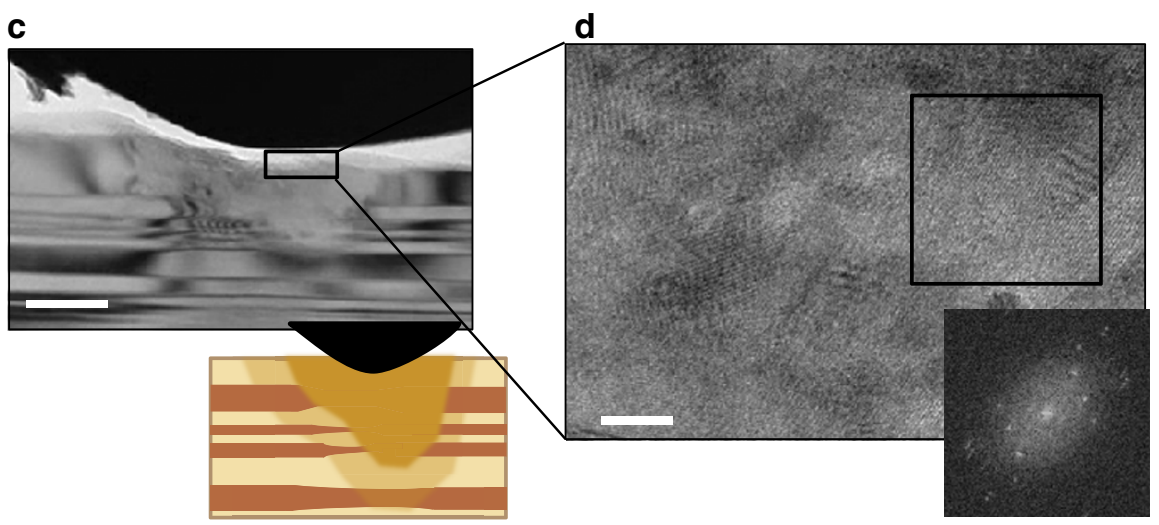

Figure 6 | Stress delocalization and energy dissipation by phase transformation in nanotwinned aragonite under nanoindentation. (a) TEM image and schematic illustration of nanoscale growth twins before nanoindentation in TEM. Scale bar, $50 \mathrm{~nm}$. (b,c) TEM images and schematic illustrations showing the evolution of the deformation zone during nanoindentation (see Supplementary Movie 7). The strain field and the deformation zone consisted of amorphous phase and nanograins are highlighted in different colours. During the stress-induced local phase transformation, the nanotwins close to the transformed region show a slight change in the TB spacing. Scale bar, $50 \mathrm{~nm}$. (d) HRTEM image of the deformation zone showing the formation of nanograins through phase transformation. A fast-Fourier transformed pattern obtained from the boxed region is shown as inset in d. Scale bar, $10 \mathrm{~nm}$.

occurs in the biogenic calcite, leading to an enhancement of energy dissipation by about an order of magnitude, compared with the single-crystalline calcite mineral. It was found that while deformation twinning is spatially localized under nanoindentation, it catalyses a variety of additional inelastic mechanisms ${ }^{32,33}$, which effectively dissipates a considerable amount of energy under predatory attacks and makes the calcite-based shells resistant to catastrophic fracture. In comparison, in the aragonite-based conch shell, the crack-blocking effect of TBs and the resulting delocalization of deformation by phase transformation are identified as the dominant toughening mechanisms. It is also interesting to note that the twins in Strombus gigas shell are inherent, about 2-20 nm thick and distribute uniformly within the basic building blocks of aragonite lamellae, while the twins in Placuna placenta shell are produced by deformation, $\sim 50 \mathrm{~nm}$ thick and localize only in the deformed or damaged zone. Therefore, in the aragonite- and calcite-based shells, the nanoscale twins (from growth or deformation twinning) play a common and crucial role in maintaining sufficient toughness to prevent fracture, but the difference in microscopic deformation mechanisms essentially reflects that nature has evolved different energydissipating strategies to synthesize bio-composites with unique mechanical properties.

During the past decade, it has been demonstrated that nanoscale twins embedded in micron- or sub-micron-sized grains of polycrystalline metals induce an exceptional combination of mechanical and physical properties including ultra-high strength and hardness, good tensile ductility and strain hardening, superior fatigue and wear resistance ${ }^{21,34-38}$. Recently, nanotwinned cubic boron nitride and diamond have been synthesized in the laboratory ${ }^{39,40}$, with unprecedented hardness and toughness. Preexisting growth twins play a key role in the mechanical properties of these man-made nanotwinned materials. For instance, in nanotwinned metals, both high strength and ductility can be attained as the TBs effectively impede dislocation motion but still allow a high density of mobile dislocations to operate in a highly organized lamellar structure $21,34-38$. Our present work has demonstrated the crucial role of biogenic growth twins in the mechanical properties of biological materials. Although the nanotwinned structure seems beneficial for both engineering and biological systems, the toughening mechanisms in biogenic nanotiwnned aragonite are distinct from those observed in manmade nanotwinned metals. For example, a recent in situ TEM study ${ }^{38}$ revealed that, in nanotwinned metal foils, dislocations are emitted from a moving crack-tip and impinge on TBs ahead of the crack, leading to the formation of dislocation walls on the TBs which become impenetrable to further dislocation motion and hinder the propagation of the crack. In contrast, the mineral in biological materials has ionic bonding and is nominally brittle, and the underlying mechanisms for energy dissipation have been identified here as crack trapping at TBs, multiple nanocracking, phase transformation and strain delocalization driven by the cracktip stress field.

In summary, the present study clearly indicates that nanoscale growth twins in Strombus gigas conch shell play a critical role in the mechanical properties of the material. In situ 
nanoindentation-based fracture experiments on both nanotwinned and twin-free specimens from Strombus gigas, nacre and single-crystalline aragonite reveal that the TBs of nanoscale growth twins can effectively block crack propagation and induce phase transformation in the vicinity of the crack-tip, leading to the delocalization of deformation and efficient energy dissipation that delay catastrophic fracture, which is corroborated by atomistic simulations and FEM modelling. The results from both experiments and simulations show that the nanotwinned microstructure improves the fracture toughness of aragonite by roughly an order of magnitude. These findings provide a fundamental understanding of the contribution of hard basic building blocks at nanoscale to fracture resistance of the overall structure, which complement our previous knowledge about the roles of soft organic content and bio-composite structure in the toughness of bio-materials. The present study may also have profound impact on the design of strong and tough biomimetic materials with minimum organic content.

\section{Methods}

Transmission electron microscopy. Dry and externally cleaned Strombus gigas conch shells were cut normal to the growth line (shell axis) to expose three macroscopic layers in the cross-section of the outer whorl. The sectioned sample was sliced and polished down to $30 \mu \mathrm{m}$ (MultiPrep System 15-2000, Allied) at $45^{\circ}$ with respect to the shell surface to expose the end face and the large face of third-order lamellae in an edge-on projection (Supplementary Fig. 1a). The sample was then thinned to less than $1 \mu \mathrm{m}$ by mechanical wedge polishing (Model 590 Tripod Polisher, South Bay) (Supplementary Fig. 1b,c). The wedge polished sample was attached to a half-cut $3 \mathrm{~mm} \mathrm{Cu}$ grid using epoxy (see, for example,

Supplementary Fig. 2d). Using an $\mathrm{Ar}^{+}$ion miller (PIPS 691, Gatan, Inc.), it was further thinned for electron transparency at liquid nitrogen $\left(\mathrm{LN}_{2}\right)$ temperature. To remove any surface damage layer that might be produced by the ion milling, low energy $\mathrm{Ar}^{+}$ion milling was carried out at $250 \mathrm{~V}$ for $10 \mathrm{~min}$ using Gentle Mill (Technoorg Linda). Finally, thin layers of carbon were coated on both sides of the TEM sample to make it conductive to the electron beam. The TEM specimens of nacre and single-crystalline aragonite were prepared following the same method. A field-emission TEM (JEM-2100F, JEOL) operated at $200 \mathrm{kV}$ and a high voltage TEM (JEM-1300S, JEOL) operated at 1.25 MV were used for the conventional microstructure analysis and the atomic structure of nanoscale twins and the fractured surfaces, respectively.

In situ fracture testing in TEM. Notched TEM samples for CTOD measurement were prepared using a focused ion beam (FIB, Helios Nano-Lab, FEI). To precisely control the loading axis for in situ TEM nanomechanical testing, the samples cut from Strombus gigas conch shell and single crystal aragonite were polished to expose a specific crystallographic orientation before FIB processes. In the case of Strombus gigas conch shell, the polishing was conducted along the large face of the third-order

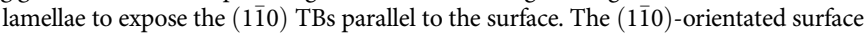
of aragonite single crystal was prepared for comparison. A thin lamella was milled using a high energy $\mathrm{Ga}^{+}$ion beam in FIB and lifted out from the polished surface and attached to a $\mathrm{Cu}$ grid through $\mathrm{Pt}$ deposition. Subsequently, the sample design shown in Supplementary Fig. 3b was patterned in top-view using a beam current of $93 \mathrm{pA}$ at $30 \mathrm{kV}$. Fine machining was carried out in side view using a beam current of $48 \mathrm{pA}$ at $30 \mathrm{kV}$ (Supplementary Fig. 3f). As a final step, low energy milling was performed at beam voltages of 5 and $1 \mathrm{kV}$ to remove surface damages and/or contamination caused by the high energy $\mathrm{Ga}^{+}$ion beam. The details on sample preparation are summarized in Supplementary Fig. 3. In situ fracture tests with CTOD measurement were carried out in a TEM (JEM-2100F, JEOL) operated at $200 \mathrm{kV}$ by using a single tilt nanoindentation holder (Nanofactory). The holder is equipped with a flat-ended diamond indenter and a piezo-stage to which a TEM specimen is mounted for precise positioning and quantitative manipulation of displacement. The diamond indenter was machined using FIB to fit into the notch of the TEM sample. The sample was mounted to the piezo-stage with great care to reduce the misalignment between the tip and the sample. All the mechanical tests were performed in displacement control mode at a displacement rate of $3 \mathrm{~nm} \mathrm{~s}^{-1}$. Dynamic events of crack initiation and propagation were recorded in real-time by using a charge-coupled device camera (ORIUS 200D, Gatan) at 25 frames per second. For in situ nanoindentation tests in TEM, the wedge-polished TEM samples and a sharp diamond indenter were used. The TEM specimen was bonded to a Au wire using silver epoxy and assembled into the piezo-stage of the nanoindentation holder. After making contact between the sample edge and the indenter tip, the indentation test was conducted at a typical displacement rate of $10 \mathrm{~nm} \mathrm{~s}^{-1}$.

FEM calculation of J-integral according to CTOD. Two-dimensional samples (Supplementary Fig. 21) were constructed in ABAQUS following the same loading and geometrical condition as in the in situ TEM experiments. Aragonite was modelled as a homogeneous elastic material with Young's modulus of $120 \mathrm{GPa}$ and Poisson's ration of 0.3 . The pre-existing crack was set to different lengths according the CTOD measurements. The J-integral value was calculated after loading until the CTOD in FEM matched with the experiment data.

Atomistic simulations. Three aragonite samples with controlled twin orientations were used for the simulations: a twin-free single-crystalline aragonite in $T$-orientation, a twin-free aragonite in $M$-orientation and a nanotwinned aragonite with alternating $T$ - and $M$-orientations (referred to as nanotwinned aragonite) in which TBs are slanted with respect to an advancing crack along a cleavage plane. Each sample is about $50 \mathrm{~nm}$ wide, $100 \mathrm{~nm}$ long and $1.1 \mathrm{~nm}$ thick and contains about 0.5 million atoms. All the MD simulations were performed using software package LAMMPS ${ }^{41}$. Interatomic forces in aragonite are described by an empirical potential ${ }^{42}$, which includes Coulomb, LJ, harmonic angular and dihedral interactions. All the simulated samples were initially relaxed and equilibrated at $5 \mathrm{~K}$ for 50 ps using Nosé-Hoover thermostat and barostat ${ }^{43}$. Periodic boundary condition is imposed along the thickness direction of the samples. After relaxation, the mode I loading is applied at a constant strain rate of $\sim 2 \times 10^{8} \mathrm{~s}^{-1}$. During loading, an NVT ensemble with Nosé-Hoover thermostat ${ }^{43}$ is used to maintain a constant temperature. Visualization is processed via software package Ovito ${ }^{44}$.

\section{References}

1. Meyers, M. A., Chen, P. Y., Lin, A. Y. M. \& Seki, Y. Biological materials: structure and mechanical properties. Prog. Mater. Sci. 53, 1-206 (2008).

2. Smith, B. L. et al. Molecular mechanistic origin of the toughness of natural adhesives, fibres and composites. Nature 399, 761-763 (1999).

3. Kamat, S., Su, X., Ballarini, R. \& Heuer, A. H. Structural basis for the fracture toughness of the shell of the conch Strombus gigas. Nature 405, 1036-1040 (2000).

4. Mayer, G. Rigid biological systems as models for synthetic composites. Science 310, 1144-1147 (2005).

5. Ortiz, C. \& Boyce, M. C. Bioinspired structural materials. Science 319, 1053-1054 (2008).

6. Espinosa, H. D. et al. Tablet-level origin of toughening in abalone shells and translation to synthetic composite materials. Nat. Commun. 2, 173 (2011).

7. Gao, H., Ji, B., Jager, I. L., Arzt, E. \& Fratzl, P. Materials become insensitive to flaws at nanoscale: lessons from nature. Proc. Natl Acad. Sci. USA 100, 5597-5600 (2003).

8. Mutvei, H. Ultrastructural characteristics of the nacre in some gastropods. Zool. Scr. 7, 287-296 (1978).

9. Kobayashi, I. \& Akai, J. Twinned aragonite crystals found in the bivalvian crossed lamellar shell structure. J. Geol. Soc. Jpn 100, 177-180 (1994).

10. Suzuki, M., Kim, H., Mukai, H., Nagasawa, H. \& Kogure, T. Quantitative XRD analysis of $\{110\}$ twin density in biotic aragonites. J. Struct. Biol. 180, 458-468 (2012).

11. Lakes, R. Materials with structural hierarchy. Nature 361, 511-515 (1993).

12. Kamat, S., Kessler, H., Ballarini, R., Nassirou, M. \& Heuer, A. H. Fracture mechanisms of the Strombus gigas conch shell: II-micromechanics analyses of multiple cracking and large-scale crack bridging. Acta Mater. 52, 2395-2406 (2004).

13. Menig, R., Meyers, M. H., Meyers, M. A. \& Vecchio, K. O. Quasi-static and dynamic mechanical response of Strombus gigas (conch) shells. Mater. Sci. Eng. A 297, 203-211 (2001).

14. Schwalbe, K.H., Newman, Jr. J. C. \& Shannon, Jr. J.L. Fracture mechanics testing on specimens with low constraint-standardization activities within ISO and ASTM. Eng. Fract. Mech. 72.4, 557-576 (2005).

15. Menig, R., Meyers, M. H., Meyers, M. A. \& Vecchio, K. S. Quasi-static and dynamic mechanical response of Haliotis rufescens (abalone) shells. Acta Mater. 48, 2383-2398 (2000).

16. Evans, A. G. \& Fu, Y. Some effects of microcracks on the mechanical properties of brittle solids-II. Microcrack toughening. Acta Metall. 33, 1525-1531 (1985).

17. Hutchinson, J. W. Crack tip shielding by micro-cracking in brittle solids. Acto Metall. 35, 1605-1619 (1987).

18. Ortiz, M. Microcrack coalescence and macroscopic crack growth initiation in brittle solids. Int. J. Solids Struct. 24, 231-250 (1988).

19. Shum, D. K. M. \& Hutchinson, J. W. On toughening by microcracks. Mech. Mater. 9, 83-91 (1990).

20. Archer, T. D. et al. An interatomic potential model for carbonates allowing for polarization effects. Phys. Chem. Miner. 30, 416-424 (2003).

21. Lu, L., Chen, X., Huang, X. \& Lu, K. Revealing the maximum strength in nanotwinned copper. Science 323, 607-610 (2009).

22. Zhu, T., Li, J., Samanta, A., Kim, H. G. \& Suresh, S. Interfacial plasticity governs strain rate sensitivity and ductility in nanostructured metals. Proc. Natl Acad. Sci. USA 104, 3031-3036 (2007).

23. Li, X., Wei, Y., Lu, L., Lu, K. \& Gao, H. Dislocation nucleation governed softening and maximum strength in nano-twinned metals. Nature 464, 877-880 (2010). 
24. Gao, H. Application of fracture mechanics concepts to hierarchical biomechanics of bone and bone-like materials. Int. J. Fract. 138, 101-137 (2006).

25. Yao, H. \& Gao, H. Multi-scale cohesive laws in hierarchical materials. Int. J. Solids Struct. 44, 8177-8193 (2007).

26. Zhang, Z., Zhang, Y. \& Gao, H. On optimal hierarchy of load-bearing biological materials. Proc. R. Soc. B 278, 519-525 (2010).

27. Bechtle, S., Ang, S. F. \& Schneider, G. A. On the mechanical properties of hierarchically structured biological materials. Biomaterials 31, 6378-6385 (2010).

28. Sen, D. \& Buehler, M. J. Structural hierarchies define toughness and defect-tolerance despite simple and mechanically inferior brittle building blocks. Sci. Rep. 1, 35 (2011).

29. Barber, D.J. \& Wenk, H.R. Deformation twinning in calcite, dolomite, and other rhombohedral carbonates. Phys. Chem. Miner. 5, 141-165 (1979).

30. Ferrill, D. A. et al. Calcite twin morphology: a low-temperature deformation geothermometer. J. Struct. Geol. 26, 1521-1529 (2004).

31. Rybacki, E. et al. Experimental deformation of synthetic aragonite marble. J. Geophys. Res. 108, 2174 (2003).

32. Li, L. \& Ortiz, C. Pervasive nanoscale deformation twinning as a catalyst for efficient energy dissipation in a bioceramic armour. Nat. Mater. 13, 501-507 (2014).

33. Ritchie, R. O. Natural materials: armoured oyster shells. Nat. Mater. 13, 435-437 (2014).

34. Lu, L., Shen, Y. F., Chen, X. H., Qian, L. H. \& Lu, K. Ultrahigh strength and high electrical conductivity in copper. Science 304, 422-426 (2004).

35. Qin, E. W., Lu, L., Tao, N. R., Tan, J. \& Lu, K. Enhanced fracture toughness and strength in bulk nanocrystalline $\mathrm{Cu}$ with nanoscale twin bundles. Acta Mater. 57, 6215-6225 (2009).

36. Singh, A., Tang, L., Dao, M., Lu, L. \& Suresh, S. Fracture toughness and fatigue crack growth characteristics of nanotwinned copper. Acta Mater. 59, 2437-2446 (2011).

37. Jang, D., Cai, C. \& Greer, J. R. Influence of homogeneous interfaces on the strength of $500 \mathrm{~nm}$ diameter $\mathrm{Cu}$ nanopillars. Nano Lett. 11, 1743-1746 (2011).

38. Kim, S. W., Li, X., Gao, H. \& Kumar, S. In situ observations of crack arrest and bridging by nanoscale twins in copper thin films. Acta Mater. 60, 2959-2972 (2012).

39. Tian, Y. et al. Ultrahard nanotwinned cubic boron nitride. Nature 493, 385-388 (2013).

40. Huang, Q. et al. Nanotwinned diamond with unprecedented hardness and stability. Nature 510, 250-253 (2014).

41. Plimpton, S. Fast parallel algorithms for short-range molecular dynamics. J. Comp. Phys. 117, 1-19 (1995)

42. Xiao, S., Edwards, S. A. \& Grater, F. A new transferable forcefield for simulating the mechanics of CaCO3 crystals. J. Phys. Chem. C 115, 20067-20075 (2011).
43. Nosé, S. A unified formulation of the constant temperature molecular dynamics methods. J. Chem. Phys. 81, 511-519 (1984).

44. Stukowski, A. Visualization and analysis of atomistic simulation data with OVITO-the open visualization tool. Model. Simul. Mater. Sci. Eng. 18, 015012 (2010).

\section{Acknowledgements}

This work was supported by National Research Foundation of Korea (NRF) grant funded by the Korea government (NRF-2015R1A2A2A01007904, NRF-

2015M3D1A1070672), and Green Science program by the POSCO. S.Y., X.L., T.Z. and H.G. acknowledge the support by NSF through Award CMMI-1161749, and by MRSEC Program through award DMR-0520651 at Brown University, and computational support from the National Institute for Computational Sciences (NICS) through Grant MS090046. X.L. also acknowledges support by the Chinese 1000-talent plan and the NSFC grants 11372152 and 51420105001

\section{Author contributions}

S.H.O. and H.G. conceived the project. S.H.O. designed the experiments. X.L. and H.G. designed the modelling and simulations. Y.A.S., S.L., S.M., J.J. and M.K. prepared the specimens and performed the in situ TEM testing. S.Y., X.L. and T.Z. performed the molecular dynamics, finite-element, coarse-grained simulations and self-similar hierarchical modelling. All the authors analysed the data, discussed the results and wrote the paper.

\section{Additional information}

Supplementary Information accompanies this paper at http://www.nature.com/ naturecommunications

Competing financial interests: The authors declare no competing financial interests.

Reprints and permission information is available online at http://npg.nature.com/ reprintsandpermissions/

How to cite this article: Shin, Y. A. et al. Nanotwin-governed toughening mechanism in hierarchically structured biological materials. Nat. Commun. 7:10772 doi: 10.1038/ ncomms10772 (2016)

(c) (i) This work is licensed under a Creative Commons Attribution 4.0 International License. The images or other third party material in this article are included in the article's Creative Commons license, unless indicated otherwise in the credit line; if the material is not included under the Creative Commons license, users will need to obtain permission from the license holder to reproduce the material. To view a copy of this license, visit http://creativecommons.org/licenses/by/4.0/ 